\title{
Coupling between experiment and numerical simulation of shape memory alloy multicrystal
}

Tarek Merzouki ${ }^{1, a}$, Christophe Collard ${ }^{2}$, Nadine Bourgeois ${ }^{3}$, Tarak Ben zineb ${ }^{1}$, and Fodil Meraghni $^{2}$

1 Laboratoire d'Energétique et de Mécanique Théorique et Appliqué (LEMTA), Nancy-Université, CNRS, 2, rue Jean Lamour 54519 Vandoeuvre les Nancy, France

2 Laboratoire de Physique et Mécanique des Matériaux (LPMM), CNRS, Arts et Métiers ParisTech, 4 rue Augustin Fresnel, 57078 Metz, France

3 Laboratoire de Physique et Mécanique des Matériaux (LPMM), CNRS, Université Paul Verlaine de Metz, ile du Saulcy, 57045 Metz, France

\begin{abstract}
This paper deals with the experimental and numerical analysis of the effect of stress-strain heterogeneities due to elastic anisotropy, grain orientations and their effects on martensitic transformation for a Cu-Based SMA multicrystal. The shape of each grain is measured successively by optical microscopy and Electron Back Scattered Diffraction technique. During a tensile loading at room temperature, the displacement field of the free surface is measured by Digital Image Correlation. The considered shape for the finite element analysis is designed on the basis of the experimental characterization of the shape of each grain. The model is intended to simulate the behaviour of the multicrystal. The experimentally obtained data, such as the actual applied boundary conditions at the edge of the area, crystallographic orientation, and some material parameters of the literature chosen to be in conformity with our case, are introduced in the numerical model designed by the Abaqus finite element code. A SMA micromechanical constitutive law, implemented in this FE code, is considered for the behaviour description of each grain. It describes the effect of a martensitic transformation on the behaviour of a single crystal by taking into account the possible activation of various martensite variants. The study shows that the experimental and numerical results are in good agreement. Moreover, numerical results show the stress state in grains is disturbed by the neighboring grains and this disturbance has a strong influence on the martensite variant activation.
\end{abstract}

\section{Introduction}

Some Shape Memory Alloys (SMA) present a superelastic behaviour generated by a reversible austenite-martensite transformation. This phase transformation occurs at the grain scale, it can be induced by cooling or by the application of mechanical loading. While the applied stress increases, the austenite is gradually transformed in martensite, induces locally large strain and stress heterogeneities due to transformation. When the loading is removed, the reverse martensitic transformation takes place, and the initial material shape is recovered. Scale transition techniques are used for the modelling of the SMA polycrystals macroscopic behaviour takes. Models have to take into account the effect of the phase transformation. Generally, they are based on micromechanical approaches $[1,2]$ or on phenomenological ones $[3,4]$. In micromechanical models, the local thermomechanical behaviour at the grain scale is assumed to be

\footnotetext{
a e-mail: tarek.merzouki@esstin.uhp-nancy.fr
} 
homogeneous and interactions between martensite variants and between grains are taken into account through average quantities. These approaches are well adapted to the polycrystals where grain size is really small compared to the size Representative Elementary Volume (REV) and to the studied structure. In the case of the multicrystals, where the grain size is of the same order of the loaded structure dimension, it is necessary to take into account geometrical and crystallographic heterogeneities in order to simulate the macroscopic behaviour of the structure. In this study, a multicrystal subdomain of 65 grains is considered, it is the zone of interest (ZOI) in a Cu-Based SMA sample. The multicrystal is obtained from a bulk material by many successive hot rolling passes. The crystallographic orientation of each grain is determined by Electron Back-Scatter Diffraction (EBSD) technique. Then, an in-situ machine is used to carry out a tensile test on the studied sample. Simultaneously, the actual applied boundary conditions (displacements) at the edge of the ZOI are measured by Digital Image Correlation (DIC) from images of the upper free surface. Correli-Q4 [5] is adopted for correlation leading to the local displacements and hence to the strain fields. In order to setup the correspondent finite element model, a SMA singlecrystal constitutive law [1] is implemented into the finite element code Abaqus via the User MATerial (UMAT) subroutine. The equations describing the thermomechanical behaviour of the singlecrystal are based on the description of the martensitic transformation at the crystal lattice scale[6]. The analysis of the experimental and numerical results highlights the effect of the different levels of heterogeneity on the global thermomechanical behaviour. In fact, for a considered grain, the boundary conditions induced by their neighbours have a strong influence on the martensite variant activation. Moreover, inside grains, the activation of a martensite variant modifies locally the stress state and may activate one or more other variants.

\section{Experimental procedure}

The studied material is a CuAlBe SMA, the composition is with 11.5 wt. \% Al and 0.5wt. \%Be. The specimen surface preparation is carried out using a mechanical polishing on both sides of the sample, followed by an electro-polishing, and the thickness is then reduced to reach about $0.3 \mathrm{~mm}$. The specimen was etched in order to reveal the microstructure. Figure 1 exhibits the micrography of the upper side of a CuAlBe SMA obtained by optical microscopy. The sample is etched only after EBSD. The Figure indicates the numbering of the 65 grains considered in the studied area (red zone). There is only one grain in its thickness since the mean grain size is widely higher than the specimen thickness. Grain boundaries are not perfectly perpendicular to the free surface, they are slightly tilted. However, in the framework of this study, the grain edges are assumed to be normal to the specimen surface. For this material, the temperature when

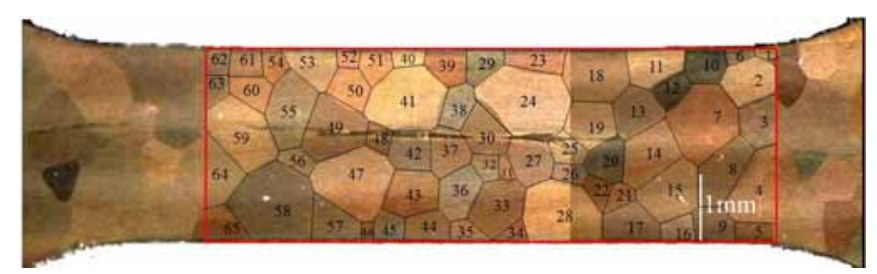

Fig. 1. Optical micrography of the upper surface of a CuAlBe SMA

the martensitic transformation starts (noted $M s$ ) was measured using the Differential Scanning Calorimetry (DSC) [7]. The authors indicated a temperature up to $-85 C^{o}$. Therefore the alloy is $100 \%$ austenitic at room temperature and stress free state. Some tensile tests carried out in the same material allow estimating the stress of the transformation at room temperature, it is between $160 \mathrm{MPa}$ and $190 \mathrm{MPa}$ [7]. Crystallographic orientation of each grain is measured by the EBSD technique. The indexing of the Kikuchi lines is carried out by the Channel5 system (HKL Technology, Denmark). The analyzed area covers almost all the calibrated zone of the 
specimen. the obtained Euler angles are given in Table 1 as a function of the grain location in the multicrystal (see Figure 1).

\begin{tabular}{|c|c|c|c|c|c|c|c|c|c|c|c|c|c|c|}
\hline Grain & $\varphi_{1}\left({ }^{\circ}\right)$ & $\phi\left({ }^{o}\right)$ & $\varphi_{2}\left({ }^{o}\right)$ & $R_{11}$ & Grain & $\varphi_{1}\left({ }^{\circ}\right)$ & $\phi\left(^{o}\right)$ & $\varphi_{2}\left({ }^{\circ}\right)$ & $R_{11}$ & Grain & $\varphi_{1}\left({ }^{o}\right)$ & $\phi\left(^{o}\right)$ & $\varphi_{2}\left({ }^{\circ}\right)$ & $R_{11}$ \\
\hline 1 & 242.3 & 22.26 & 54.91 & 0.36 & 23 & 343.92 & 42.2 & 61.36 & 0.36 & 45 & 231.24 & 8.42 & 78.71 & 0.37 \\
\hline 2 & 341.09 & 37.14 & 17.22 & 0.5 & 24 & 267.39 & 32.84 & 64.33 & 0.29 & 46 & 47.6 & 43.8 & 48.2 & 0.45 \\
\hline 3 & 355.65 & 31.1 & 84.22 & 0.5 & 25 & 20.93 & 44.08 & 8.76 & 0.43 & 47 & 344.84 & 37.86 & 35.31 & 0.46 \\
\hline 4 & 99.33 & 27.47 & 76.38 & 0.46 & 26 & 351.26 & 17.72 & 24.28 & 0.49 & 48 & 11.71 & 45.74 & 72.82 & 0.48 \\
\hline 5 & 59.25 & 29.31 & 31.9 & 0.47 & 27 & 256.14 & 40.87 & 19.7 & 0.39 & 49 & 6.26 & 45.22 & 69.45 & 0.49 \\
\hline 6 & 294.9 & 41.1 & 52.2 & 0.41 & 28 & 7.1 & 38.7 & 24.6 & 0.45 & 50 & 266.7 & 34.92 & 20.16 & 0.38 \\
\hline 7 & 77.45 & 38.19 & 19.75 & 0.41 & 29 & 208.24 & 45.74 & 55.76 & 0.45 & 51 & 349.88 & 42.29 & 38.62 & 0.44 \\
\hline 8 & 3.73 & 22.08 & 79.23 & 0.5 & 30 & 117.64 & 22.56 & 73.57 & 0.45 & 52 & 295.62 & 47.33 & 26.98 & 0.27 \\
\hline 9 & 32.9 & 14.44 & 46.01 & 0.49 & 31 & 167.17 & 46.21 & 52.3 & 0.35 & 53 & 311.82 & 34.17 & 18.81 & 0.35 \\
\hline 10 & 64.29 & 45.69 & 31.02 & 0.39 & 32 & 26.68 & 33.42 & 27.11 & 0.35 & 54 & 33.95 & 51.88 & 45.84 & 0.37 \\
\hline 11 & 228.71 & 30.79 & 50.11 & 0.48 & 33 & 6.23 & 32.87 & 51.19 & 0.43 & 55 & 205.47 & 16.12 & 62.45 & 0.5 \\
\hline 12 & 307.9 & 27.3 & 46.1 & 0.49 & 34 & 0.1 & 49.9 & 50.4 & 0.39 & 56 & 137.35 & 41.18 & 87.87 & 0.25 \\
\hline 13 & 344.71 & 20.97 & 17.04 & 0.49 & 35 & 257.4 & 30.3 & 46.6 & 0.25 & 57 & 44.3 & 14.9 & 1.6 & 0.33 \\
\hline 14 & 270.34 & 46.38 & 28.82 & 0.31 & 36 & 123.19 & 38.57 & 46.17 & 0.44 & 58 & 51.97 & 15.04 & 75.68 & 0.37 \\
\hline 15 & 156.64 & 34.02 & 7.22 & 0.47 & 37 & 71.78 & 42.5 & 2.2 & 0.32 & 59 & 67.26 & 42.44 & 13.29 & 0.36 \\
\hline 16 & 250.05 & 32.42 & 6.1 & 0.4 & 38 & 272.24 & 14.79 & 82.52 & 0.49 & 60 & 170.93 & 46.55 & 55.45 & 0.38 \\
\hline 17 & 336.37 & 18.85 & 49.27 & 0.46 & 39 & 34.76 & 42.3 & 44 & 0.41 & 61 & 160.85 & 35.88 & 57.61 & 0.35 \\
\hline 18 & 29.81 & 31.69 & 80.89 & 0.43 & 40 & 190.27 & 39.2 & 61.22 & 0.48 & 62 & 167.2 & 36.7 & 9.2 & 0.5 \\
\hline 19 & 12.55 & 38.06 & 22.26 & 0.43 & 41 & 199.26 & 36.05 & 77.78 & 0.5 & 63 & 184.1 & 36.1 & 14.8 & 0.49 \\
\hline 20 & 174.77 & 7.23 & 6.88 & 0.48 & 42 & 160.1 & 24.4 & 66.1 & 0.36 & 64 & 185.51 & 33.52 & 86.09 & 0.49 \\
\hline 21 & 152.16 & 44.03 & 37.4 & 0.43 & 43 & 111.7 & 49.93 & 43.42 & 0.34 & 65 & 19.37 & 43.88 & 50.33 & 0.43 \\
\hline 22 & 152.16 & 44.03 & 37.4 & 0.43 & 44 & 354.77 & 30.32 & 66.16 & 0.46 & & & & & \\
\hline
\end{tabular}

Table 1. Crystallographic orientation of the grains given as a function of their location (see Figure 1). Euler angles are defined by $\left(\varphi_{1}, \phi, \varphi_{2}\right)$. The component $R_{11}$ of the Schmid tensor, given in the loading direction

\section{Finite element modelling of SMA multicrystal}

The considered constitutive law was implemented in the finite element code Abaqus by Collard et $a l$. in framework of its work analysis of precipitate effects on shape memory alloys behaviour [6], and is based on the code Simula+ [8]. The model is based on the description of the thermomechanical behaviour of a SMA singlecrystal governed by the martensitic transformation. It is induced by a thermomechanical loading and depends on the lattice structure and orientation. The implementation procedure is based on the User MATerial subroutine (UMAT). All additional details concerning the model and the parameters governing the behaviour can be obtained in the thesis of Merzouki [9]. Some material characteristics have not been able to be experimentally identified, they are directly taken from the literature. These parameters are given in Table 2. The finite element model of the studied multicrystal area is presented in Figure 2. It should be noticed that only the central part of the sample described by Figure 1 (red zone) is considered for the finite element analysis. The dimensions of the modelled area are $8.34 \mathrm{~mm}$ long, $2.76 \mathrm{~mm}$ wide and $0.27 \mathrm{~mm}$ thick. The studied area consists of 65 grains with only one grain through the thickness. During modelling two hypotheses are adopted, (I) the grain boundaries are assumed to be normal to the specimen surface so as to simplify the FE discretization, (II) the grain shape is considered polygonal. Each grain is meshed with hexahedral (C3D8) and tetrahedral (C3D6) solid continuum isoparametric elements with linear interpolation. These elements have selective integration method corresponding to a complete integration of the deviatoric tensor and a reduced integration of the hydrostatic one. Three elements are introduced along the thickness although only one grain is considered according to this direction. The sample is meshed with 12294 elements. The loading is carried out at constant temperature. Only the superelastic behaviour is exploited. This finite element model is considered for the simulation of multicrystal behaviour under tensile loading.

It should be noticed that the modelled area is located inside the zone fixed between the two grips of the tensile machine; the boundary conditions must be adapted to reproduce the real 
loading as well as possible. For that, two variable displacements are applied to each node of the left and right edges of the simulated area in the 1 and the 2-direction (respectively loading and transverse direction). The in-plane field displacements are obtained experimentally by the Image Correlation technique on the two sections of the simulated area and used as applied boundary conditions. The displacement is assumed uniform through thickness. Nodal FE positions do not necessarily correspond to the measured ones. Consequently, a linear interpolation is carried out between measured points leading to the field of displacement across the edges. In order to avoid rigid body motion, one node is fixed in the third direction (direction of thickness). By applying the experimental displacement at each node of the two edges, it is possible to take into account the interaction of the neighbouring grains. The variation in the time of the boundary conditions is supposed linear.

\begin{tabular}{ccccc}
\hline$B\left(\mathrm{MPa} /{ }^{\circ} \mathrm{C}\right)$ & $M s\left({ }^{\circ} \mathrm{C}\right)$ & $A f\left({ }^{\circ} \mathrm{C}\right)$ & $\mathrm{g}$ & \\
\hline 0.2 & -85 & -70 & 0.23 & \\
\hline Compatible interaction (H1) & Incompatible interaction $(\mathrm{H} 2)$ & $C_{11}(\mathrm{GPa})$ & $C_{12}(\mathrm{GPa})$ & $C_{44}(\mathrm{GPa})$ \\
\hline$\mu / 350$ & $\mu / 70$ & 142.38 & 95.24 & 124.1 \\
\hline
\end{tabular}

Table 2. Material parameters and elastic constants used in the code $[10,11,7]: C_{11}, C_{12}$ and $C_{44}$ are the elasticity constants, $\mu$ is the shear modulus defined as a function of $C_{11}, C_{12}$ and $C_{44}, M s$ is the martensite start temperature, $A f$ is the austenite finish temperature, $B$ is the stress-temperature coefficient, (H1) and (H2) are two parameters used in the constitutive model

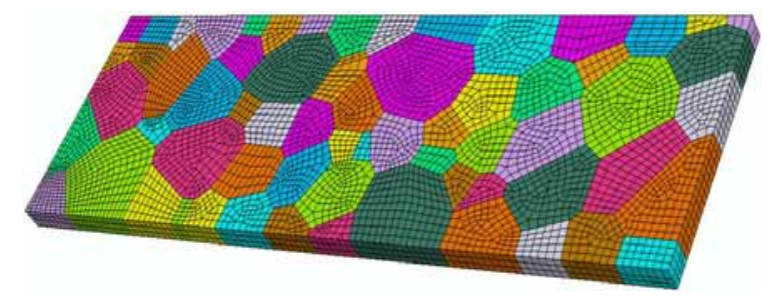

Fig. 2. FE model of the CuAlBe SMA

\section{Results and analysis}

Figure 3.a presents the global behaviour of the multicrystal and is represented by expressing the global stress versus the global strain. The global stress is calculated from the resultant of the reactions in one section nodes according to the loading direction, whereas the global strain is obtained from the average elongation in the loading direction. Figure 3.b shows that the evolution of the global volume fraction of martensite versus the global strain is almost linear. The transformation appears for a loading corresponding to about $0.25 \%$ of global longitudinal strain. Below that strain, the behaviour of the multicrystal is elastic, which is confirmed by the curve of the global behaviour.

Figure 4 presents the local behaviour of some grains and is defined from the equivalent Von Mises stress and strain averaged on all the elements belonging to the considered one. For a loading level corresponding to $2 \%$ of global longitudinal strain, well oriented grains compared to loading direction present a higher strain level. As an example, local strain value reaches $8 \%$ for grains $(18,19,20$ and 22). For a better understanding of the transformation evolution, the average volume fraction of martensite in each grain is expressed as a function of the global longitudinal strain, as defined in figure 4. The martensitic fraction generally presents a higher value in the well oriented grains $(R 11>0.4)$. As an example, grains 20 and 22 . It should be 


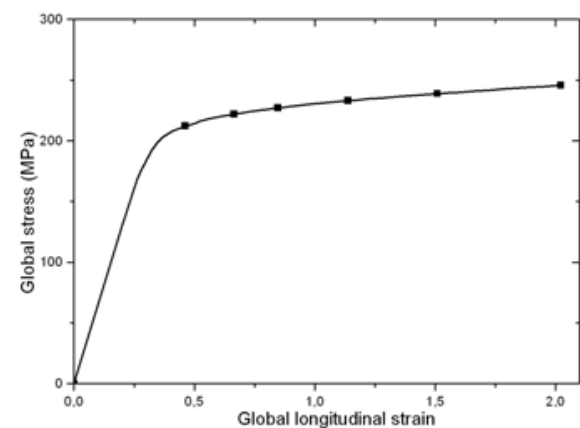

(a) Global behaviour

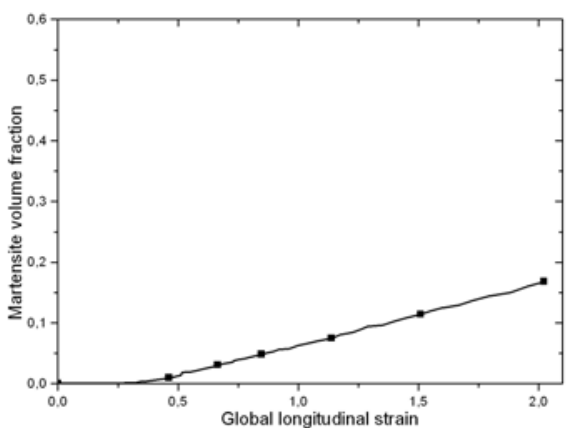

(b) Global volume fraction of martensite

Fig. 3. Global behaviour and martensitic transformation of the multicrystal

noted that for a global strain of about $1 \%$, some grains are close to a $20 \%$ volume fraction of martensite (grains 20,22. etc), whereas other grains are always in an austenitic state (grains 23, 24. etc), so their behaviour remain elastic. At a $2 \%$ of global strain, the global volume fraction of martensite in the whole sample is about $15 \%$ when it approaches $50 \%$ in the grains 20 and 22. Some grains have rather close crystallographic orientations so the pseudoelastic part of the behaviour is quasi-identical. A rather weak strain is observed, as an example in grains 33 and 36 even if they are rather well oriented compared to the loading direction. This is due to the neighbouring grains which prevent them from becoming more deformed.
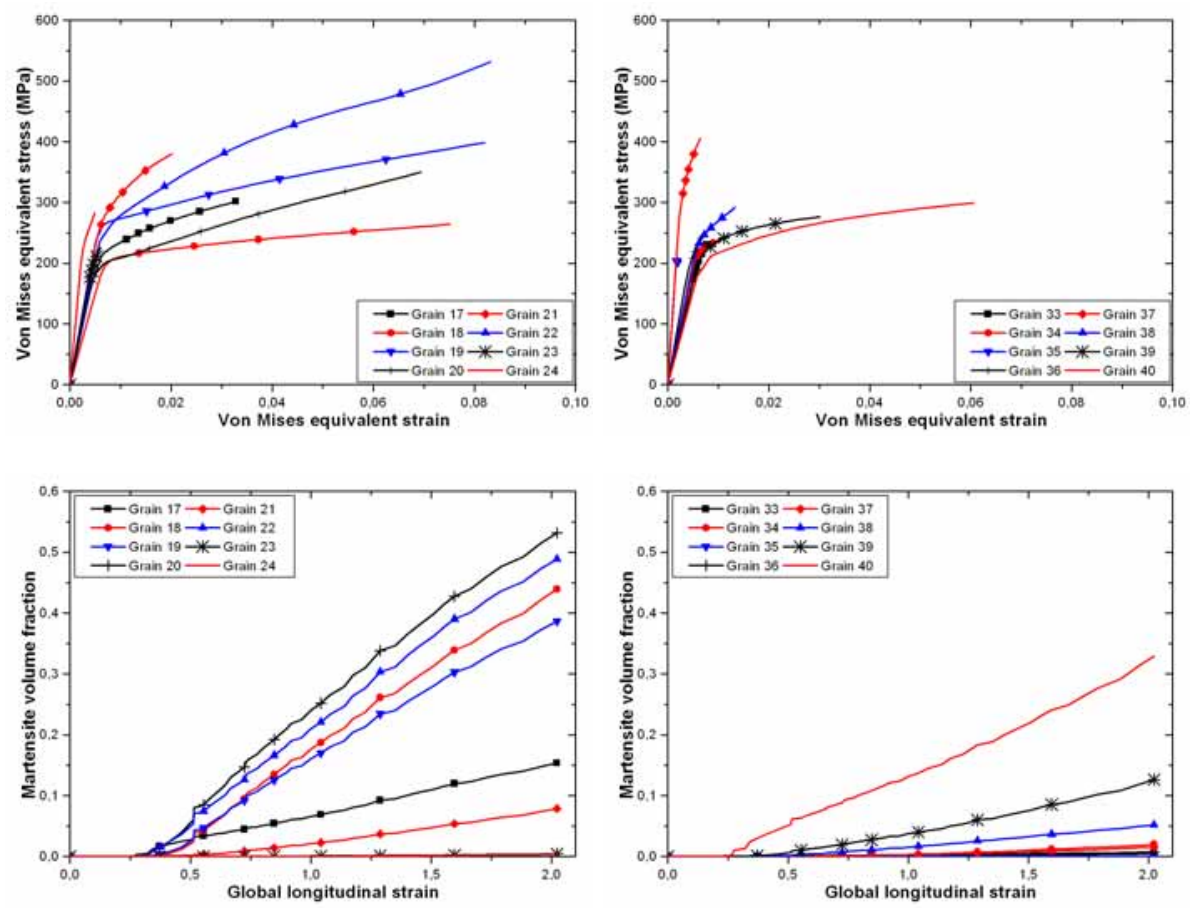

Fig. 4. Local behaviour and martensitic transformation of some grain of the multicrystal 


\section{Qualitative comparison of martensitic transformation}

Figure 5 shows a comparison between experimental results and numerical calculation carried out by applying experimental boundary conditions BCs measured by image correlation, in terms of martensitic transformation distribution at the loading corresponding to $0.8 \%$ of global longitudinal strain. The simulated transformation distribution is similar to the one observed experimentally. These results confirm the importance to apply the experimental BCs. Moreover, a grain located at the boundary is not experimentally deformed. This can only be obtained by applying the experimental BCs. Such procedure takes into account the interaction of the neighbouring grains. This coupling between the experimental measurements and FE simulation allows simulating a realistic local and global mechanical response via the interactions between the grains. So it is necessary to apply this type of BCs to simulate the behaviour of small-size structures. However, the evolution of imposed displacement at each node during the loading is hardly known. For small loadings, the measured displacements may not be evaluated since they are lower than the experimental errors.

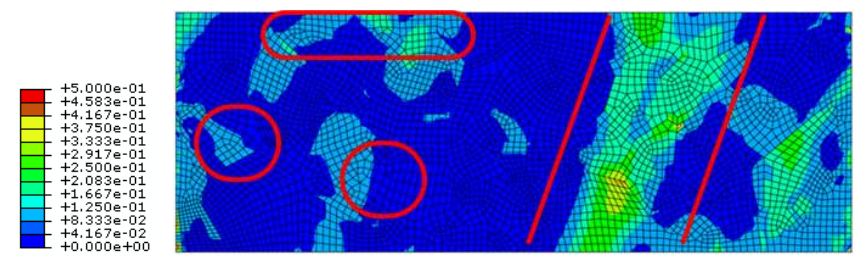

(a) Numerical calculation

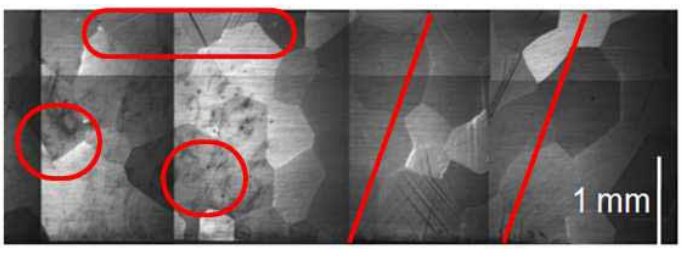

(b) Experimental observation

Fig. 5. Comparison of the martensitic transformation

\section{Conclusion}

The confrontation between numerical calculation and experimental analysis for a SMA multicrystal is detailed in this study. On the one hand, we have seen how the experiment provided certain essential information to the simulation of the multicrystal behaviour and on the other hand, we have observed that the experiment could be reproduced by the simulation. The shapes of the grains observed in optical microscopy is reproduced on the grid finite elements. Only one grain is considered in the thickness. The shape of each grain is defined as a subdomain and meshed by three dimensional continuum elements. The boundary conditions BCs of the free surface are measured by Digital Image Correlation. A SMA singlecrystal constitutive model is implemented into the finite element code Abaqus. The qualitative comparison between experiment and the FE simulations carried out by applying experimental BCs, in terms of martensitic transformation distribution is discussed. The description of the grain boundaries and the mesh refinement is led to numerical results which are in a good qualitative agreement with experimental findings. It has been shown that the transformation localization domain predicted by the FE simulations has the same spatial repartition than the one observed experimentally. The results show the importance to apply the experimental BCs. Indeed, the coupled experimentalnumerical approach highlighted that the stress-strain state in such grains is disturbed by the 
other grain neighbours. The developed approach is used to assess the effects of this stress-strain disturbance on the martensite variant activation.

\section{Acknowledgements}

The constitutive equation for the single crystal behavior are solved by the Simula + scientific computing code. It is developed at the Laboratoire de Physique et Mécanique de Matériaux (LPMM), Metz, France, and at the Laboratoire de Mathématiques et ses Applications de Valenciennes (LAMAV), Valenciennes, France.

\section{References}

1. E. Patoor and M. Berveiller. Micromechanical modeling of thermoelastic behavior of shape memory alloys. in CISM lecture notes number 368 Mechanics of Solids with phase change, edited by F. D. Fischer and M. Berveiller, 368:121-188, 1997.

2. L.C. Brinson. Simplification and comparisons of shape memory alloy constitutive models. Journal of Intelligent Materials Systems and Structures, 7:108-114, 1996.

3. D. C. Lagoudas and Z. Bo. Thermomechanical modeling of polycrystalline smas under cyclic loading, part ii: material characterization and experimental results for a stable transformation cycle. International Journal of Engineering Science, 37:1141-1173, 1999.

4. C. Lexcellent, S. Leclercq, B. Gabry, and G. Bourbon. The two way shape memory effect of shape memory alloys: an experimental study and a phenomenological model. International Journal of Plasticity, 16:1155-1168, 2000.

5. F. Hild and S. ROUX. CorreliQ4: A software for -finite-element- displacement field measurements by digital image correlation. Internal report no. 269, LMT-Cachan, 2002.

6. C. Collard, T. Ben-Zineb, E. Patoor, and M.O. Ben Salah. Micromechanical analysis of precipitate effects on shape memory alloys behaviour. Materials Science and Engineering: A, 481-482:366-370, 2008.

7. B. Kaouache, S. Berveiller, K. Inal, A. Eberhardt, and P. Patoor. Stress analysis of martensitic transformation in cualbe polycrystalline and single-crystalline shape memory alloy. Materials Science and Engineering A, 378:232-237, 2004.

8. Simula + : is a scientific computing code. it is developed at the laboratoire de physique et mécanique de matériaux (lpmm), metz, france, and at the laboratoire de mathématiques et ses applications de valenciennes (lamav), valenciennes, france. http://www.univvalenciennes.fr/lamav/Simula $+/$.

9. T. Merzouki. Experimental identification and micromechanical modelling of the behaviour of a multicrystal in shape memory alloy. Phd thesis, Arts et Mtiers ParisTech, 2008.

10. D. Entemeyer, E. Patoor, A. Eberhardt, and M. Berveiller. Strain rate sensitivity in superelasticity. International Journal of plasticity, 16:1269-1288, 2000.

11. C. Niclaeys, T. Ben-Zineb, S. Arbab Chirani, and E. Patoor. Determination of the interaction energy in the martensitic state. International Journal of plasticity, 18:1619-1647, 2002 . 